\title{
Determination of axonal transport velocities via image cross- and autocorrelation
}

\author{
Oliver Welzel · Daniel Boening • Armin Stroebel • \\ Udo Reulbach · Jurgen Klingauf · Johannes Kornhuber • \\ Teja Wolfgang Groemer
}

Received: 12 February 2009/Revised: 31 March 2009/ Accepted: 7 April 2009

(C) European Biophysical Societies' Association 2009

\begin{abstract}
On their way to the synapse and back, neuronal proteins are carried in cargo vesicles along axons and dendrites. Here, we demonstrate that the key parameters of axonal transport, i.e., particle velocities and pausing times can be read out from CCD-camera images automatically. In the present study, this is achieved via cross- and autocorrelation of kymograph columns. The applicability of the method was measured on simulated kymographs and data from axonal transport timeseries of mRFP-labeled synaptophysin. In comparing outcomes of velocity determinations via a performance parameter that is analogous to the signal-to-noise ratio (SNR) definition, we find that outcomes are dependent on sampling, particle numbers and signal to noise of the kymograph. Autocorrelation of individual columns allows exact determination of pausing time populations. In contrast to manual tracking, correlation does not require experience, a priori assumptions or disentangling of individual particle trajectories and can operate at low SNR.
\end{abstract}

O. Welzel and D. Boening contributed equally to this work.

Electronic supplementary material The online version of this article (doi:10.1007/s00249-009-0458-5) contains supplementary material, which is available to authorized users.

O. Welzel · A. Stroebel · U. Reulbach · J. Kornhuber ·

T. W. Groemer $(\bowtie)$

Department of Psychiatry and Psychotherapy,

University of Erlangen-Nuremberg,

Schwabachanlage 6, 91054 Erlangen, Germany

e-mail: teja.groemer@uk-erlangen.de

D. Boening $\cdot$ J. Klingauf

Department of Cellular Biophysics, University of Munster,

Robert Koch Str. 31, 48149 Munster, Germany
Keywords Kymograph · Fluorescence · Vesicles · Velocities · Camera

\section{Introduction}

Axonal transport is a highly regulated process that provides the basis for replenishing synaptic function and has been subject to intensive research (Salinas et al. 2008; and for a current review see: Roy et al. 2005). Analysis of fluorescence image time series plays an increasing role. In wide field fluorescence microscopy, axonal transport particles seem to move along a defined line due to limited spatial resolution. This makes it possible to simplify the timedependent intensity information along this line of interest to a kymograph image. In this projection, location is plotted versus time, so that all movements of the particles during the recording are displayed as individual diagonal lines, their slope corresponding to their velocity. The advantage of an axonal kymograph over movies is that it constitutes a single image that contains all movement information, which can be inspected and compared conveniently at a glance (Miller and Sheetz 2004, 2006). Axonal transport can be roughly described by a small set of parameters: direction of the movement (de Wit et al. 2006), velocity of particles (Roy et al. 2007, 2008) and pausing times (Sabo et al. 2006) of particles. In most studies, axonal movement analysis is, if described, mostly done by manually tracing trajectories (Sabo et al. 2006; DeBolt et al. 2007; Niwa et al. 2008; Wang et al. 2007; Zhou et al. 2001) or possibly via particle trackers operating with the image stack (Konzack et al. 2007). However, these methods require high signal-to-noise ratio (SNR) and/or manual input. It has been shown recently that axonal transport can be measured using a two-photodiodes-based 
fluorescence cross-correlation (Stagi et al. 2005) assay. Adjustment of probe and detectors is important and acquired data are limited to two representative points of the axon. Recently, the field of fluorescence correlation spectroscopy has been extended to camera images as their acquisition rates have increased to the kilohertz level. This allows measuring diffusion constants in living cells with spatial resolution (Kannan et al. 2006, 2007). We have now established camera-based cross- and autocorrelation for the measurement of velocities and pausing times of axonally transported organelles. For direct comparison with recent studies we analyzed axonal transport by means of the well characterized fluorescently labeled synaptic vesicle protein, synaptophysin (SYP). SYP is localized to presynaptic boutons (Roy et al. 2007) and widely used as a synaptic marker. The four-transmembrane protein, which takes about $10 \%$ of the total synaptic vesicle protein (Takamori et al. 2006) moves in the fast component (Elluru et al. 1995) of axonal transport. Recent findings indicate that SYP is required for correct targeting of other synaptic vesicle proteins (Bonanomi et al. 2007). SYP undergoes both anterograde and retrograde axonal transport. In developing neurons, synaptic vesicle protein transport vesicles moving in the anterograde direction are far more frequent than those moving retrogradly (Roy et al. 2007).

\section{Materials and methods}

\section{Cell culture and transfection}

Hippocampal neuronal cultures were prepared from newborn rats as described (Klingauf et al. 1998). Newborn rats were killed by decapitation in accordance with the guidelines of the State of Bavaria. Hippocampi were removed from the brain in icecold Hank's salt solution, and after "unrolling" the dentate gyrus was cut away. After digestion with trypsin $(5 \mathrm{mg} / \mathrm{ml})$ cells were triturated mechanically and plated in MEM medium, supplemented with $10 \%$ fetal calf serum and 2\% B27 supplement (all from Invitrogen). Experiments were performed between 10 and 15 days in vitro.

On day three, neurons were transfected with SYP-mRFP under control of a CMV promoter (pcDNA-3 clontech) with calcium phosphate as described (Wienisch and Klingauf 2006).

\section{Imaging}

Experiments were conducted at room temperature on inverted microscopes (Axiovert 135, Carl Zeiss and TIEclipse, Nikon) equipped with a $63 \times, 1,2 \mathrm{NA}$ water immersion objective and a $60 \times, 1,2 \mathrm{NA}$ water immersion objective, respectively. Fluorescent dyes were excited at $550 \mathrm{~nm}$ (Polychrom II monochromator, Till Photonics; Nikon Intensilight C-HGFI), emission light was reflected on a $560 \mathrm{~nm}$ dichroic mirror (Semrock) and projected onto a cooled CCD-camera (PCO-Sensicam, Roper; iXon ${ }^{\mathrm{EM}}$ DU-885, Andor). Coverslips were placed into a perfusion chamber $($ volume $=500 \mu \mathrm{l})$ containing a modified Tyrode solution (in mM: $140 \mathrm{NaCl}, 5 \mathrm{KCl}, 2 \mathrm{CaCl}_{2}, 2 \mathrm{MgCl}_{2}, 30$ glucose, 10 Hepes, $\mathrm{pH} 7.4 ; 350 \mathrm{mOsm})$. Images were recorded with $200 \mathrm{~ms}$ exposure times at 0.5 and $2 \mathrm{~Hz}$ frame rates, respectively, using a camera with $2 \times 2$ or without binning, and converted into tagged image files. Sampling of moving subresolution transport organelles (diameter $80-150 \mathrm{~nm}$ ), with the PCO-Sensicam at $2 \times 2$ binning and $210 \mathrm{~nm}$ effective pixel size $(6.45 \times 6.45 \mu \mathrm{m}$ pixels $)$ was measured with 2.9 pixels per FWHM-PSF. For the iXon ${ }^{\mathrm{EM}}$ DU-885 $(8 \times 8 \mu \mathrm{m}$ pixels $)$, no binning yields a camera pixel in object space of $133 \mathrm{~nm}$ and 6.4 pixels per FWHMPSF. Kymographs were generated using the dropdown menu "kymograph" in MetaMorph (Molecular Devices). Only straight axonal segments were selected for analysis to avoid velocity artefacts originating from form factors such as strong axon covings.

\section{Simulation}

\section{Kymographs}

Simulations and analyses were performed using customwritten MATLAB (Mathworks) programs. Simulations of kymographs from camera image stacks were generated from single traces corresponding intensity profiles along a virtual line of interest. To generate profiles with known SNR that closely match the camera data, values of simulated particle peaks were added to Poisson noise with mean $\lambda=30$ (Sbalzarini and Koumoutsakos 2005) background. The point spread Bessel function (PSF) of the optical system of the microscope was approximated by a Gaussian profile for each subresolution particle as described elsewhere (Hassler et al. 2005). For linear trajectories, the position of the mean coordinate of each Gaussian profile in line $(x)$ is calculated from its position in the previous line $\left(x_{0}\right)$ and the specific velocity of the particle $(v)$ by $x=x_{0}+v t$, where $t$ is the time interval between two lines. Individual trajectories were added for simulation of more than one particle.

Pausing particles in kymographs were simulated with stationary Gaussians of constant amplitudes; e.g., (50, 60, 40) extending over a predefined number of rows, e.g., $(50,20,80)$ in an image of size $20 \times 100$. As aforementioned, Poisson noise $(\lambda=30)$ was added to the matrix. 
Data analysis

\section{Cross-correlation}

Velocities were determined via pairwise cross-correlation of kymograph columns. The maximum's index in the cross-correlation function (CCF) corresponds to the best overlap $\left(t_{\max }\right)$ of both columns, i.e., time vectors. Velocities are drawn directly from the so determined time and given (position of the columns) distance information $\left(d_{x, y}\right)$ :

$v=\frac{d_{x, y}}{t_{\max }}$.

In detail, kymographs were segmented into a number of squares $\left(N_{\mathrm{s}}\right)$ of typically $20 \times 20$ or $30 \times 30$ to reduce particle number per detection area (see Fig. 2). For each part of the kymograph, calculation of velocities was performed according to the following scheme (see supplemental Fig. 2): First, each column time vector with length $N_{y}$ was cross-correlated with each of all other columns in the segment. After subtraction of the median from the two examined columns, the cross-correlation is calculated by:

$c c_{x y}(\mathrm{~m})=\sum_{i=1}^{N_{y}} x_{i} \cdot y_{i+m}$,

where $x$ and $y$ are the two examined column vectors and $m$ is the shift of column $y$ compared to $x$. Note that neighbor columns have to be extended with Poisson noise estimated from the kymograph data $(\lambda=$ median(kymograph)), to obtain the length $2 N_{y}$. This operation is indispensable for accurate calculation of velocities via cross-correlation, as it keeps the number of CC summands constant. Thus assuming Poisson noise, the probability of observing a maximum at a time-shift $(m)$ is equally distributed in $\left\{1 \ldots N_{y}\right\}$. One property of the cross-correlation $\left(c c_{x y}\right)$ is that the discrete time-shift $(m)$ directly corresponds with the best overlap $\left(t_{\max }\right)$, so that the velocity $(v)$ can be calculated with Eq. 1. Next, we increased the performance of CC verifying the authenticity of the detected maxima using tests for false positives and significance. False-positive maxima are generated by the particle PSF extension over adjacent columns. They can be sorted out by reverse correlation of the columns. If the maximum is then found at the same place with a higher value of CCF than the one calculated before, the particle is considered to move in the opposite direction and $t_{\max }$ is discarded. The verification is performed in a column environment sized to the variance of the assumed Gaussian PSF approximation. To ensure the authenticity of the maximum in CCF, we used Grubb's test for outliers with a significance level of 0.05 . Using both verification tests the performance (see below) of CC velocity determinations is increased by a factor greater than 10 (see supplemental Fig. 4). When each column is correlated with each other in both directions the maximum number of velocities $\left(N_{\mathrm{v}}\right)$ is given by:

$N_{\mathrm{v}}=N_{s} 2\left(\begin{array}{c}N_{x} \\ 2\end{array}\right)$

and thus depends directly on the number of columns $\left(N_{x}\right)$ and the number of squares $\left(N_{\mathrm{s}}\right)$. Therefore the number of successfully determined velocities changes with the lateral size of the kymograph. The number of equally determined velocities is counted to create a histogram. Velocities greater or less than 1 pixel/frame occur in less columns than the velocity of $1 \mathrm{pixel} / \mathrm{frame}$. To obtain absolute comparable counts of the determined velocities, this discretization phenomenon is adjusted by weighting all counted velocities with an adjustment-factor for count correction. Each adjustment factor depends only on the size of the squares $\left(N_{\mathrm{s}}\right)$. For example, a particle moving with the velocity of 2 pixel/frame can maximally occur in every second column. Thus, to achieve comparablility to the counted velocitiy of $1 \mathrm{pixel} / \mathrm{frame}$, the velocity of $2 \mathrm{pixel} /$ frame is multiplied with 2 .

\section{Autocorrelation}

For the determination of pausing times, the autocorrelation function (ACF) is calculated from individual kymograph columns after median subtraction by:

$a c_{x x}(m)=\sum_{i=1}^{N_{y}-m} x_{i} \cdot x_{i+m}$

Here, $x$ is the examined column vector and $m$ is the shift of column $x$ with itself. When simplified, pausing particles display rectangular profiles in the column vector. Thus, autocorrelation functions of pausing particles show a linear decay to zero, of which the bend point at the time axis corresponds to the pausing time (Fig. 3b). The automated detection of the bend point is hindered by its position near the noise level. We thus extrapolated the bend point positions from the index of the ACF decay to $50 \%$ of its initial amplitude.

\section{Performance}

To examine the quality and influence of kymograph parameters on the $\mathrm{CC}$ method, we defined a performance parameter $(p)$ in analogy to the SNR definition as the ratio of the root-mean square (RMS) of the number of the correctly identified velocities $\left(n_{\mathrm{v}, \mathrm{c}}\right)$ to the RMS of the number of all other determined velocities $\left(n_{\mathrm{v}, \mathrm{o}}\right)$ : 
$p=\left(n_{\mathrm{v}, \mathrm{c}}^{-1} \sum_{i=1}^{n_{\mathrm{v}, \mathrm{c}}} v_{\mathrm{i}, \mathrm{c}}^{2}\right)^{0.5}\left(n_{\mathrm{v}, \mathrm{o}}^{-1} \sum_{i=1}^{n_{\mathrm{v}, \mathrm{o}}} v_{\mathrm{i}, \mathrm{o}}^{2}\right)^{-0.5}$

where $v_{\mathrm{i}, \mathrm{c}}$ are correctly identified velocities and $v_{\mathrm{i}, \mathrm{o}}$ are other velocities. If no other velocities are found, $p$ is infinity and decreases hyperbolically to zero as more of the other velocities are found.

\section{Results}

Cross-correlation velocity determination (CCVD)

As a starting point, we simulated kymographs with known particle velocities for proof of principle (Fig. 1 a). After positive preliminary tests with binary kymographs (data not shown), we decided to simulate typical camera image stack-derived kymographs more realistically (compare Fig. 1a + detail in $\mathrm{c}$ ) including the particles point spread functions and Poisson noise. This had the advantage of immediate adaptability to real data that we acquired in video-microscopy of SYP-mRFP axonal transport in rat hippocampal neurons (Fig. 1c-e). Vectors drawn from cross-correlation of kymograph columns show prominent peaks on the best overlap in time $\left(t_{\max }\right)$ accessible by automatic detection (Fig. 1b, and "Materials and methods"). As the positions of the columns are known, velocities can be drawn directly with $t_{\max }$ and displayed in a histogram (Fig. 1b, inset). We next probed CCVD on kymographs from axonal transport SYP-mRFP (Fig. 1c, see also supplemental Fig. 3 for another example). Correlation of small segments exactly resembled the situation in the preceding simulations (Fig. 1c detail $+\mathrm{d}$ histogram). When whole kymographs are correlated (Fig. 1c), multiple velocity populations occur in the histogram (Fig. 1e).

Validation via noise replacement

We next sought to consolidate findings from CCVD by comparing its results with our subjective impression. For this, we replaced visually identified trajectories in the kymographs with Poisson noise (see supplemental Fig. 1af). This enabled us to assign trajectory parts to velocity populations in the histogram (arrows in Fig. 1c). We were able to show that individual velocity populations originate from defined trajectories (see supplemental Fig. 1a-f). Thus results from CCVD fit the subjective visual impression. Noise replacement was also used to validate pausing time determinations (see below).

\section{Performance testing}

To measure the general applicability, we defined a performance parameter $p$ (Eq. 5) in analogy to the SNR definition with correctly determined velocities integrated in the "signal" part and other velocities in the "noise" part.
Fig. 1 Determination of transport velocities from a kymograph via column-wise cross-correlation (CC) in simulated and real data. a Simulated particle trajectory kymograph $(\mathrm{SNR}=1.1)$ and b its normalized CC profiles (mean given as bold black trace). All CC profiles were aligned for the well-defined automatically detectable peak. Inset shows the corresponding velocity histogram of analyzed columns. c Kymograph from an image time series $(0.5 \mathrm{~Hz})$ of a hippocampal axon, SYP labeled with mRFP. d Histogram of calculated velocities from detail and e total kymograph show that velocity populations (identified via noise replacement, see text) can be clearly defined (arrows). Asterisks are defined in Fig. 3
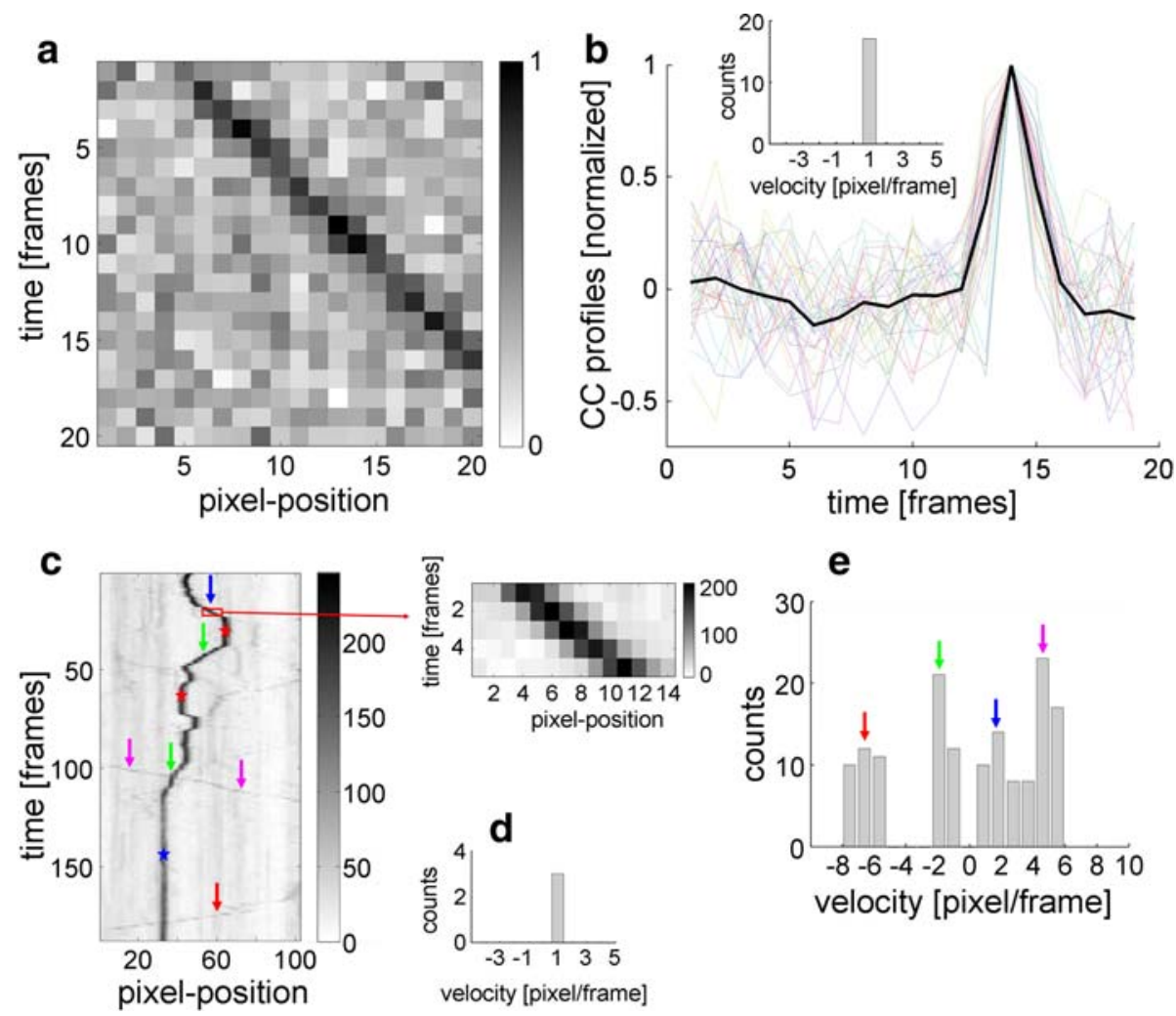
As a ratio, $p$ gets values between zero if the correct velocity is never detected and infinity for perfect determination. For the simulation, we generated sets of kymographs with random trajectories in which any trajectory starting point was allowed. This leads to varying performance levels, e.g., when a particle just crosses the edge of the image. In this case, even when its velocity is detected correctly, its contribution to the performance level is minor as its number of columns is small compared to the total number of columns. As expected, CCVD performance increased with SNR (Fig. 2a), but decreased with higher values of the sampling, i.e., the ratio of particle size to kymograph width (Fig. 2b). The performance decreased with a higher number of particles (Fig. 2c) and can be improved via segmentation of the kymograph. This means that the kymograph is segmented into smaller ones, where the particle number is reduced ideally to one particle per detection area. The segmentation is limited by sampling (Fig. 2b) and the resolution of the possible velocities, which are determined by the detection size. Hence, an optimal segmentation can be found (Fig. 2d), which depends, however, on the desired resolution. The frequency of the detectable velocities differs for diverse velocities, e.g., the velocity 2 (pixel/frame) is only detectable half the time as the velocity 1 (pixel/frame). Because this ratio is predefined by the size of the kymograph, it can be easily compensated. At this point, however, it is important to underline that the velocity histogram determined by CCVD is still qualitatively correct with a high reliability, but, as mentioned above, not quantitatively. Nevertheless, histograms from different experiments can be compared.

\section{Determination of pausing times}

In a simulated kymograph, three predefined pausing times of 20,50 and 80 frames could be determined correctly via column-wise autocorrelation (ACPTD) (Fig. 3a-c, and detailed in methods). We observed minor differences between preset and determined pausing times in columns at the PSF border (Fig. 3c). These can be attributed to faster decaying ACF at worse SNR at the borders. After the simulation, we calculated axonal pausing times of SYPmRFP by autocorrelation. Analogous to the velocity analysis, we observed different populations in the histogram (Fig. 3d) that we could assign to visible pauses in the kymograph (asterisks in Figs. 1c, 3d) via noise replacement as stated above. We showed all determined pausing times instead of filtering the result, for example by cutting off extreme values or by only using AC amplitudes over a well-defined threshold. Populations around ten frames are shown in more detail in the inset of Fig. 3d. Around 15 frames, a population can be clearly identified that corresponds to the marked pausing particle (Fig. 1c). Another example is given in supplemental Fig. 3, in which additional distinct pausing populations of around 30 and $60 \mathrm{~s}$ can be seen. Very long pausing times, which correspond to
Fig. 2 Influence of kymograph parameters on the performance (p) Eq. 2 of CC velocity detection from random trajectories. The performance $p$ is not only dependent on SNR a, but also on the relation between particle size and kymograph width, i.e., the sampling $\mathbf{b}$. As with increasing number of particles $p$ decreases $\mathbf{c}$ at higher particle numbers, the performance can be improved via segmentation, which reduces particle numbers per detection area, but at small areas is limited by the sampling so that an optimal segmentation $\mathbf{d}$ can be found. Kymograph sizes were $20 \times 20(2 \mathrm{a}-\mathrm{c})$ and $100 \times 100$, respectively. $n=20(\mathbf{a}-\mathbf{c})$ and $n=10(\mathbf{d})$ iterations. All error bars indicate SEM
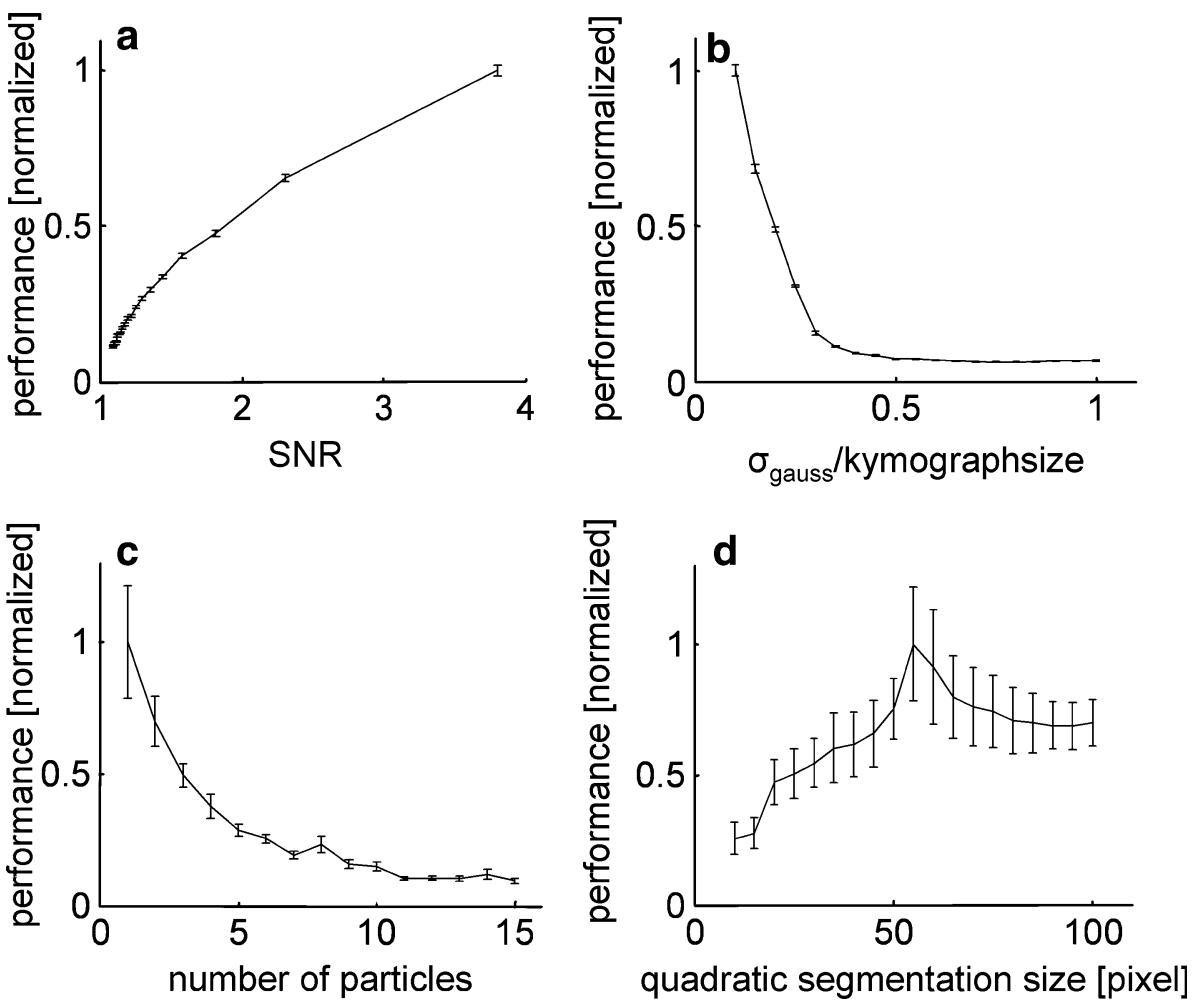
Fig. 3 Determination of pausing times from a kymograph via column-wise autocorrelation (AC) in simulated and real data. a Simulated particle trajectory kymograph and $\mathbf{b}$ its $\mathrm{AC}$ profiles. All AC profiles, which are calculated from a column with a pausing particle, show linear decay. The time till the bend point of the AC profiles is proportional to the pausing time of the particle. Pausing time histogram from simulated data c and from video-microscopy (Fig. 1c) d. Pausing time populations can be clearly defined. Corresponding pauses (validated via noise replacement, see text) in the particle trajectory are indicated by asterisks (see Fig. 1c). Inset shows pausing times from 0 to 20 frames with higher number of bins

the vertical lines in the kymograph at about 5 and $85 \mu \mathrm{m}$ were also identified (pausing times above $85 \mathrm{~s}$ ).

\section{Discussion}

Tracing single trajectories on kymograph images has predominated the analysis of transport vesicle movement on axons. In this procedure, the observer can be very sensitive if experienced, but hardly impartial. Of course, the analysis by experienced human specialists will outperform pattern detection algorithms in most cases. However, selection criteria in trajectory definition if done by human subjects will hardly be exactly defined, and practicable data sets will be limited in amount or by "operator fatigue". We show that image correlation methods can be applied in the analysis of key transport parameters, i.e., velocities and pausing times from kymograph images. Based on video-microscopy data, axonal transport velocities can be read out from kymographs via CCVD. CCVD results in amounts of velocities that can be grouped into a histogram. As CCVD does not depend on a priori information or experience, it allows for direct comparison between different measurements of axonal transport velocities (see supplemental Fig. 1) and opens the way to fully automated data analysis. Besides demonstrating its principle, we tested CCVD for its applicability by simulating realistic trajectory data. We find that CCVD is able to operate even at SNR as low as 1 . This is confirmed by the observation that CCVD can
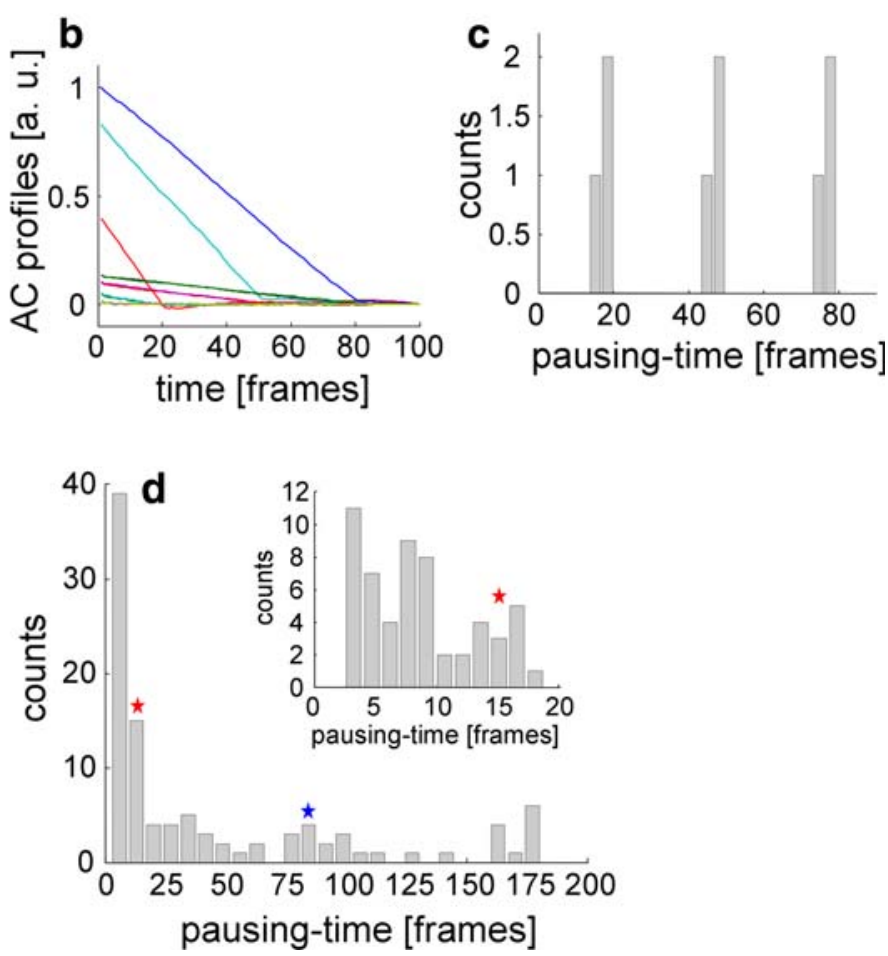

determine velocities from simulated trajectories that were difficult to detect visually. We next showed that pausing times of transport vesicles can be resolved via autocorrelation-based pausing time determination (ACPTD). As we could show in simulations and kymograph trajectories of SYP-mRFP, ACPTD is precise and does not require arbitrary pausing time definitions (Sabo et al. 2006). Different pausing time populations can clearly be distinguished. CCVD is restricted to the trajectory-independent overall analysis of movement. This is both the advantage and the disadvantage of the method. On the one hand, it can produce information on the velocities of the fluorescent proteins even when the trajectories can hardly be traced manually. On the other hand, it cannot be used to measure the behavior of single transport vesicles. These characteristics are also true for ACPTD. Correlation of image information enlarges the scientists' toolbox for the automated analysis of dynamic processes, minimizes observer errors and thus allows screening.

\section{References}

Bonanomi D, Rusconi L, Colombo CA, Benfenati F, Valtorta F (2007) Synaptophysin I selectively specifies the exocytic pathway of synaptobrevin 2/VAMP2. Biochem J 404:525-534. doi: 10.1042/BJ20061907

de Wit J, Toonen RF, Verhaagen J, Verhage M (2006) Vesicular trafficking of semaphorin $3 \mathrm{~A}$ is activity-dependent and differs between axons and dendrites. Traffic 7:1060-1077. doi: 10.1111/j.1600-0854.2006.00442.x 
DeBolt S et al (2007) Morlin, an inhibitor of cortical microtubule dynamics and cellulose synthase movement. Proc Natl Acad Sci USA 104:5854-5859. doi:10.1073/pnas.0700789104

Elluru RG, Bloom GS, Brady ST (1995) Fast axonal transport of kinesin in the rat visual system: functionality of kinesin heavy chain isoforms. Mol Biol Cell 6:21-40

Hassler K, Anhut T, Rigler R, Gosch M, Lasser T (2005) High count rates with total internal reflection fluorescence correlation spectroscopy. Biophys J 88:L01-L03

Kannan B et al (2006) Electron multiplying charge-coupled device camera-based fluorescence correlation spectroscopy. Anal Chem 78:3444-3451. doi:10.1021/ac0600959

Kannan B et al (2007) Spatially resolved total internal reflection fluorescence correlation microscopy using an electron multiplying charge-coupled device camera. Anal Chem 79:4463-4470. doi:10.1021/ac0624546

Klingauf J, Kavalali ET, Tsien RW (1998) Kinetics and regulation of fast endocytosis at hippocampal synapses. Nature 394:581-585. doi: $10.1038 / 29079$

Konzack S, Thies E, Marx A, Mandelkow EM, Mandelkow E (2007) Swimming against the tide: mobility of the microtubule-associated protein tau in neurons. J Neurosci 27:9916-9927. doi: 10.1523/JNEUROSCI.0927-07.2007

Miller KE, Sheetz MP (2004) Axonal mitochondrial transport and potential are correlated. J Cell Sci 117:2791-2804. doi:10.1242/ jcs. 01130

Miller KE, Sheetz MP (2006) Direct evidence for coherent lowvelocity axonal transport of mitochondria. J Cell Biol 173:373381. doi:10.1083/jcb.200510097

Niwa S, Tanaka Y, Hirokawa N (2008) KIF1Bbeta- and KIF1Amediated axonal transport of presynaptic regulator Rab3 occurs in a GTP-dependent manner through DENN/MADD. Nat Cell Biol 10:1269-1279. doi:10.1038/ncb1785

Roy S, Zhang B, Lee VM, Trojanowski JQ (2005) Axonal transport defects: a common theme in neurodegenerative diseases. Acta Neuropathol 109:5-13. doi:10.1007/s00401-004-0952-x
Roy S, Winton MJ, Black MM, Trojanowski JQ, Lee VM (2007) Rapid and intermittent cotransport of slow component-b proteins. J Neurosci 27:3131-3138. doi:10.1523/JNEUROSCI.499906.2007

Roy S, Winton MJ, Black MM, Trojanowski JQ, Lee VM (2008) Cytoskeletal requirements in axonal transport of slow component-b. J Neurosci 28:5248-5256. doi:10.1523/JNEUROSCI. 0309-08.2008

Sabo SL, Gomes RA, McAllister AK (2006) Formation of presynaptic terminals at predefined sites along axons. J Neurosci 26:1081310825. doi:10.1523/JNEUROSCI.2052-06.2006

Salinas S, Bilsland LG, Schiavo G (2008) Molecular landmarks along the axonal route: axonal transport in health and disease. Curr Opin Cell Biol 20:445-453. doi:10.1016/j.ceb.2008.04.002

Sbalzarini IF, Koumoutsakos P (2005) Feature point tracking and trajectory analysis for video-imaging in cell biology. J Struct Biol 151:182-195. doi:10.1016/j.jsb.2005.06.002

Stagi M et al (2005) Breakdown of axonal synaptic vesicle precursor transport by microglial nitric oxide. J Neurosci 25:352-362. doi: 10.1523/JNEUROSCI.3887-04.2005

Takamori S et al (2006) Molecular anatomy of a trafficking organelle. Cell 127:831-846. doi:10.1016/j.cell.2006.10.030

Wang X, Shaw WR, Tsang HT, Reid E, O'Kane CJ (2007) Drosophila spichthyin inhibits BMP signaling and regulates synaptic growth and axonal microtubules. Nat Neurosci 10:177185. doi:10.1038/nn1841

Wienisch M, Klingauf J (2006) Vesicular proteins exocytosed and subsequently retrieved by compensatory endocytosis are nonidentical. Nat Neurosci 9:1019-1027

Zhou HM, Brust-Mascher I, Scholey JM (2001) Direct visualization of the movement of the monomeric axonal transport motor UNC-104 along neuronal processes in living Caenorhabditis elegans. J Neurosci 21:3749-3755 